\title{
Text Similarity Measurement Using Concept Representation of Texts
}

\author{
Abhinay Pandya ${ }^{1}$ and Pushpak Bhattacharyya ${ }^{2}$ \\ 1 DA-IICT, Gandhinagar \\ abhinay_pandya@da-iict.org \\ 2 Dept. of CSE, IIT Bombay \\ pb@cse.iitb.ac.in
}

\begin{abstract}
Measuring semantic nearness of documents is important for accurate information retrieval, automated text categorization and classification. Inspired by the observation that text documents contain semantically coherent set of ideas/topics, this paper presents the design and experimental evaluation of a method to represent a text document as a set of concepts. Based on this, we propose a method to measure semantic nearness of texts. Our method makes use of WordNet which is a lexico-semantic network of words. We bypass word sense disambiguation. In order to show the effectiveness of our representation of texts, we compare experimental results of text classification and clustering with the results of classification and clustering with standard techniques.
\end{abstract}

\section{Introduction}

A key-step towards achieving the goal of retrieving all and only the most relevant information, is detecting semantic similarity, i.e., judging how similar the contents of the two texts are. 1] has given an information theoretic definition of similarity. Fundamentally, the similarity of two objects is measured by the number of features they have in common. In contemporary IR, the words of a text are considered to be their features regardless of their meaning, and the similarity of texts is estimated based on the number of words they have in common (TF) and the relative importance of these in the corpus (IDF). Unfortunately, owing to the richness (and vagaries) of natural languages such as English, these methods fail to identify the actual semantic relatedness between texts if words from both texts are matched directly.

Many approaches have been proposed in the past to measure similarity between words based on their relationships such as synonymy-antonymy, hypernymyhyponymy, meronymy-holonymy ${ }^{1}$, etc. 9] 6] [4]. But these require finding the correct sense of each word in the text- Word Sense Disambiguation (WSD)- which in itself is a difficult problem to solve. Evidently, we need a method to represent a text in terms of its semantic features even when accurate sense information

\footnotetext{
${ }^{1}$ Hypernymy: is-a relationship, hyponymy: opposite of it, meronymy: has-a relationship, holonymy: part-of relationship.
} 
of the words in the text is not available. Latent Semantic Indexing (LSI) [5] attempts to capture semantic information by observing co-occurrence of words. However, this will fail if semantically related words do not co-occur frequently.

Semantic similarity between two texts is perceived based on the commonness of the concepts contained in them. We propose a representation of a text as a set of concepts discussed in it. Similarity between two texts with this representation can then be estimated based on the semantic relatedness ${ }^{2}$ of the concepts they describe.

Some works of relevance are mentioned now. [7] presents a method to represent text in the form of WordNet 2] hypernyms and shows improved performance for the classification task. 8] shows improvement in clustering task with synset representation of texts. These methods ignore all relationships between synsets other than hypernymy and relies on the WordNet structure without adapting it to the corpus (i.e., non-information-theoretic).

In the next section we describe our methodology of text representation. Section 3 describes the experimental setup and the results. Section 4 concludes the paper.

\section{Conceptual Representation of Texts}

Finding a set of synsets as a representation of text is trivial if the correct sense (image of a word in the WordNet, synset) of each word in the given text is known. In the absence of WSD, we let each word in a given text map to all of its possible images in the WordNet and discard those synsets that are semantically very far from other synsets. Such synsets, which are far from the others, either do not represent the correct sense of words or are not representative of a given text.

Many approaches have been presented in the past to measure the semantic distance between synsets in the WordNet [9, [4, etc. [6]'s measure combines link-distance and information theoretic methods and also takes into account the type of relationship between synsets, depth of the synsets in the WordNet, and density of the region around the synsets. Based on these, a weight is attached to the edge between a child node $c$ and its parent node $p$ :

$$
w t(c, p)=\left(\beta+(1-\beta) \frac{\bar{E}}{E(p)}\right)\left(\frac{d(p)+1}{d(p)}\right)^{\alpha}[I C(c)-I C(p)] T(c, p)
$$

where $d(p)$ denotes the depth of the node $p$ in the hierarchy, $E(p)$ the number of edges in the child links, $\bar{E}$ the average density in the whole hierarchy, and $T(c, p)$ the link relation/type factor such as hypernymy/hyponymy, meronymy/holonymy, etc. The parameters $\alpha(\alpha \geq 0)$ and $\beta(0 \leq \beta \leq 1)$ control the degree of how much the node depth and density factors contribute to the edge weighting calculation. Node density in different parts of the hierarchy

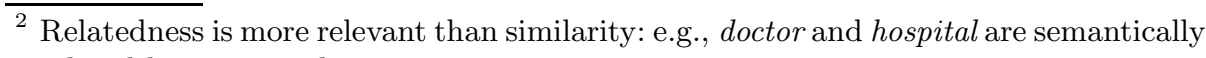
related but not similar. 
is different, and greater density around a node (e.g., plant/flora section of WordNet) indicates more 'closeness'. Information content (IC) values for synsets can be calculated as follows:

$$
I C(c)=-\log P(c)
$$

where $I C(c)$ is the information content value of synset $c$ and $P(c)$ is the probability of encountering an instance of synset $c . P(c)$ can be calculated by finding the relative frequency of synset $c$ in a sense-tagged corpus such as SemCor [3], in which each word is tagged with a WordNet synset identifier.

The overall distance between two nodes $c_{1}$ and $c_{2}$ would thus be the summation of edge weights (calculated as shown in equation (1)) along the shortest path linking two nodes:

$$
\operatorname{dist}\left(c_{1}, c_{2}\right)=\sum_{c \in\left\{\operatorname{path}\left(c_{1}, c_{2}\right)\right\}} w t(c, \operatorname{parent}(c))
$$

The above measures rely only on the WordNet structure for semantic relatedness between concepts and do not take into account their pragmatic relationships. For example, the similarity between concepts doctor\#1 and hospital\#2 would be estimated lower than what actually is with all the above methods. We attempt to capture pragmatic associations between concepts by their gloss definitions in the WordNet. Very often the glosses of related concepts have a few words in common (see [10]). The word medical is in the intersection of glosses of doctor\#1 and hospital\#2. Our measure of similarity between two concepts is as follows:

$$
\text { concept_sim }\left(c_{1}, c_{2}\right)=\frac{1}{\operatorname{dist}\left(c_{1}, c_{2}\right)} \times \delta \times \text { gloss_intersect }\left(c_{1}, c_{2}\right)
$$

where $\delta(0 \leq \delta \leq 1)$ controls the weightage of gloss_intersection in the similarity measurement.

\subsection{Semantic Vicinity}

With the enhanced notion of similarity between WordNet synsets as described above, we define semantic vicinity of a synset $s$ as a set of all synsets that are within a specified link distance from $s$. Each node in the semantic vicinity of $s$ is assigned a score (weight value) indicating its degree of relatedness with $s$. In order to select only the relevant concepts from the text, our method starts with building semantic vicinities for each input synset as described in the procedure (see algorithm 1). $M A X$ controls the definition of similarity by specifying the maximum radius of influence of similarity measure. The more the value of $M A X$, the more is the dilution of similarity. Input to our algorithm is a list of unique nouns since nouns are the least polysemous compared to other parts of speech ${ }^{3}$ and therefore believed to carry the main burden of expression. In addition to nouns, we consider denominal verbs, adjectives and adverbs that have only one sense $^{4}$. For all practical IR applications, these POS are as informative as nouns.

\footnotetext{
${ }^{3}$ From http://www. cogsci.princeton.edu/ wn/man/wnstats.7WN.html

${ }^{4}$ This is achieved by following the "DERIVATION" pointer from the synset structures in WordNet.
} 
We now present the algorithm as follows:

Data : list of unique nouns, denomial verbs, adjectives, and adverbs Result : semantic vicinities corresponding to all senses of all input nouns foreach noun $n_{i}$ in the noun-list do

foreach sense $s_{j}$ of noun $n_{i}$ do for $L=0$ to $M A X$ do for all synsets $k_{L}$, if $k_{L} \triangleleft s_{j}$ or $s_{j} \triangleleft k_{L}$, then $\operatorname{SNet}\left(s_{j}\right)=\operatorname{SNet}\left(s_{j}\right) \cup$ $\left\{k_{L}\right\}$ where the symbol $\triangleleft$ between $k_{L}$ and $s_{j}$ denotes any of the following relationship types:

- hypernymy: $k_{L}$ is-a-kind-of $s_{j}$

- hyponymy: $s_{j}$ is-a-kind-of $k_{L}$

- meronymy: $s_{j}$ has-a $k_{L}$

- holonymy: $k_{L}$ has-a $s_{j}$

- antonymy: $k_{L}$ is-opposite-of $s_{j}$

- gloss-overlap: $k_{L}$ 's gloss contains one or more words that are also a part of $s_{j}$ 's gloss.

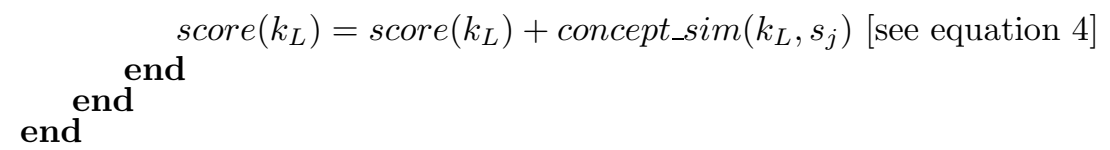

Algorithm 1. Procedure for building semantic vicinities

Representative synsets of a text among all synsets for a text can be found by discarding those synsets that are semantically distant from the rest. This is achieved by finding the intersection of semantic vicinities found in the algorithm above and selecting only those synsets that belong to majority of the semantic vicinities.

\section{$3 \quad$ Experiments}

\subsection{Classification}

250 documents in each of the categories comp.graphics, rec.autos, rec.sport.hockey, rec.sport.baseball from the 20Newsgroup dataset (a collection of 20,000 USENET articles) were chosen for our classification experiments. Table 1 summarizes the results obtained when the following classification algorithms were run on our representation: Naive Bayesian, $k-N N$ and $S V M$. For Naive Bayesian and $k$-NN, Rainbow utility provided as part of the Bow toolkit ${ }^{5}$ was used and for SVM, $S V M^{\text {light6 }}$ was used.

The results are reported with the parameters that obtained the best performance. The first row shows the results obtained with the TFIDF vector representation of the documents. The second row displays the results when the

\footnotetext{
5 http://www-2.cs.cmu.edu/ $\sim_{\text {mccallum/bow/ }}$

6 http://svmlight.joachims.org/
} 
Table 1. Comparison of performance of classification experiments on 20 Newsgroup data

\begin{tabular}{|l|l|l|l|}
\hline & Naive Bayesian & $k$-NN (k=35) & SVM (rbf, $\gamma=1.1)$ \\
\hline TFIDF, simi funct unchanged & 0.98 & 0.98 & 0.66 \\
\hline CFICF, simi funct unchanged & 0.88 & 0.80 & 0.77 \\
\hline bag-of-concepts, simi funct as Eq 5 & - & 0.87 & 0.76 \\
\hline
\end{tabular}

documents are treated as bag-of-concepts. In this case, the similarity function in the classification algorithms is kept unchanged; only the representation of the documents is changed from TFIDF to CFICF (concept frequeny/inverse concept frequency). The third row of the table shows the results when the similarity function in each of the techniques was replaced with the following similarity function:

$$
\operatorname{sim}\left(D_{1}, D_{2}\right)=\frac{\text { concept_set_sim }\left(D_{1}, D_{2}\right)}{\sqrt{\text { concept_set_sim }\left(D_{1}, D_{1}\right) \times \text { concept_set_sim }\left(D_{2}, D_{2}\right)}} \times 100 \%
$$

where concept_set_sim $(A, B)=\sum_{c_{1} \in A, c_{2} \in B}$ concept_sim $\left(c_{1}, c_{2}\right)$ and concept_sim $\left(c_{1}, c_{2}\right)$ can be calculated as shown in equation (4).

\subsection{Clustering}

The clustering experiments are performed with the same dataset as above. We used the Cluto ${ }^{7}$ library for clustering. With Repeated Bisection and I2 criterion function, TFIDF-based clustering achieved 0.91 Fscore; whereas clustering based on conceptual representation of text (our method) achieved $\mathbf{0 . 7 9}$.

Table 2. Results of clustering experiments with 20 Newsgroup dataset

\begin{tabular}{|l|l|l|}
\hline & 4 clusters & 3 clusters \\
\hline TFIDF (F-score) & 0.97 & 0.57 \\
\hline our method (F-score) & 0.79 & 0.61 \\
\hline
\end{tabular}

\section{Discussions, Conclusions and Future work}

The reason for the higher score of TFIDF on both classification and clustering is the fact that replies to postings often include the original message in them, thereby duplicating words and increasing intra-class similarity based on TFIDF measure. The reasons for lower accuracy in cases where our approach is used are (i) shortness of articles in newsgroup articles, (ii) the fact that a small set of words are frequently used, and (iii) inaccurate IC values resulting from the smallness of the SemCor corpus. However, as evident from table 2 finding 3

\footnotetext{
7 http://www-users.cs.umn.edu/ karypis/cluto/
} 
clusters from the 4 categories of news items chosen will put rec.sport.hockey and rec.sport.baseball in the same cluster by our method. Lacking common set of frequent words, TFIDF-based measures cannot achieve this 'generalization'.

These observations are promising and confirm that text similarity detection should be tackled from all three directions using lexical, syntactic and semantic knowledge.

In future we would like to investigate if retrieval performance can be improved by bridging a query and a candidate document through WordNet with its conceptual representation.

\section{References}

1. Dekang Lin: Information Theoretic definition of similarity. Proc. 15th International Conf. on Machine Learning, 1998.

2. Christiane Fellbaum: WordNet, An Electronic Lexical Database. The MIT press, 1999.

3. Francis and Kucera: Computational Analysis of present day American English. Brown University press, 1967.

4. Philip Resnik: Semantic Similarity in a Taxonomy: An Information-Based Measure and its Application to Problems of Ambiguity in Natural Language. Journal of Artificial Intelligence Research (JAIR), 11: 95-130, 1999.

5. Michael W. Berry, Susan T. Dumais, Gavin W. O'Brien: Using Linear Algebra for Intelligent Information Retrieval. SIAM Review 37:4, 1995

6. Jiang and Conrath: Semantic similarity based on Corpus statistics and lexical Taxonomy. Proceedings of International Conference Research on Computational Linguistics, 1997.

7. Sam Scott and Stan Matwin: Text classification using WordNet hypernyms. Proc. of the COLING/ACL Workshop on Usage of WordNet in Natural Language Processing Systems, 1998.

8. Sam Scott and Stan Matwin: WordNet improves text document clustering. In Proc. of the Semantic Web Workshop at SIGIR-2003", 2003.

9. R. Rada and H. Milli and E. Bicknell and M. Blettner : Development and Application of a Metric on Semantic Nets. IEEE Transactions on Systems, Man and Cybernetics, vol. 1, no. 9: 17-30, 1989.

10. Michael Lesk: Automatic sense disambiguation: How to tell a pine cone from an icecream cone. In Proc. of the 1986 ACM SIGDOC conference, pages 24-26, New York. 